Supporting Information

\title{
Structure-Property Relationships of Poly(glycolic acid) and poly(2-hydroxybutyrate)
}

\author{
Yuji Sasanuma,* Hiromi Yamamoto, and Somin Choi \\ Department of Applied Chemistry and Biotechnology, Graduate School and \\ Faculty of Engineering, Chiba University \\ 1-33 Yayoi-cho, Inage-ku, Chiba 263-8522, Japan
}

Formulation A. Statistical Weight Matrices, $U_{j}$ 's ( $j$ : bond number), of Poly(glycolic acid)

For the bond designation, see Figure 1 . The $U_{j}$ matrices of the first unit are

$$
\begin{aligned}
& U_{1}=U_{\mathrm{a} 1}=C_{1} \otimes R_{1} \\
& U_{\mathrm{b} 1}=C_{1} \otimes R_{1} \otimes R_{3}
\end{aligned}
$$

and

$$
U_{\mathrm{c} 1}=\left(\begin{array}{ccccccccc}
\mathrm{t} & \mathrm{s} & & \mathrm{t} & \mathrm{s} & & \mathrm{t} & \mathrm{s} & \\
\mathrm{t} \mathrm{t} & \mathrm{t} \mathrm{g}^{+} \\
0 & 0 & 0 & u_{\mathrm{tg}+\mathrm{t}} & u_{\mathrm{tg}+\mathrm{s}} & 0 & 0 & 0 & 0 \\
u_{\mathrm{ttt}} & u_{\mathrm{tts}} & 0 & 0 & 0 & 0 & 0 & 0 & 0 \\
0 & 0 & 0 & 0 & 0 & 0 & u_{\mathrm{tg}-\mathrm{t}} & u_{\mathrm{tg}-\mathrm{s}} & 0 \\
0 & 0 & 0 & 0 & 0 & 0 & 0 & 0 & 0 \\
0 & 0 & 0 & 0 & 0 & 0 & 0 & 0 & 0 \\
0 & 0 & 0 & 0 & 0 & 0 & 0 & 0 & 0 \\
0 & 0 & 0 & 0 & 0 & 0 & 0 & 0 & 0 \\
0 & 0 & 0 & 0 & 0 & 0 & 0 & 0 & 0 \\
0 & 0 & 0 & 0 & 0 & 0 & 0 & 0 & 0
\end{array}\right)
$$

where $\otimes$ stands for the direct product,

$$
\begin{gathered}
C_{1}=\left(\begin{array}{l}
1 \\
0 \\
0
\end{array}\right) \\
R_{1}=\left(\begin{array}{lll}
1 & 0 & 0
\end{array}\right) \\
R_{3}=\left(\begin{array}{lll}
1 & 1 & 1
\end{array}\right)
\end{gathered}
$$

and, for example,

$$
u_{\mathrm{tg}^{+} \mathrm{s}}=\exp \left(-\Delta E_{\mathrm{tg}^{+} \mathrm{s}} / R T\right)
$$


is the statistical weight of the $\operatorname{tg}^{+} \mathrm{s}$ conformation, in which bonds $\mathrm{a}_{1}, \mathrm{~b}_{1}$, and $\mathrm{c}_{1}$ adopt trans, gauche ${ }^{+}$, synperiplanar states, respectively. $E_{\mathrm{tg}^{+} \mathrm{s}}$ is its conformational energy, $R$ is the gas constant, and $T$ is the absolute temperature.

The $U_{j}$ matrices of the subsequent repeating unit are

$$
\begin{gathered}
U_{\mathrm{a}}=C_{3} \otimes I_{3} \otimes R_{1} \\
U_{\mathrm{b}}=C_{3} \otimes C_{1} \otimes R_{1} \otimes R_{3}
\end{gathered}
$$

and

$$
U_{\mathrm{c}}=U_{c 1}
$$

where

$$
C_{3}=\left(\begin{array}{l}
1 \\
1 \\
1
\end{array}\right)
$$

and

$$
I_{3}=\left(\begin{array}{lll}
1 & 0 & 0 \\
0 & 1 & 0 \\
0 & 0 & 1
\end{array}\right)
$$

The $U_{j}$ matrices of the $m$ th and $n$th bonds are

$$
U_{\mathrm{m}}=U_{a}
$$

and

$$
U_{n}=U_{\mathrm{b}}
$$

Formulation B. Statistical Weight Matrices, $U_{j}$ 's ( $j$ : bond number), of Poly(2-

\begin{tabular}{|c|c|c|c|c|c|c|c|c|}
\hline$(1,1)$ & $(1,2)$ & $(1,3)$ & $(1,4)$ & $(1,5)$ & $(1,6)$ & $(1,7)$ & $(1,8)$ & $(1,9)$ \\
\hline $\begin{array}{l}u_{\mathrm{ttt}(\mathrm{t})} \\
(2,10)\end{array}$ & $\begin{array}{l}u_{\mathrm{ttt}\left(\mathrm{g}^{+}\right)} \\
(2,11)\end{array}$ & $\begin{array}{l}u_{\mathrm{ttt}\left(\mathrm{g}^{-}\right)} \\
(2,12)\end{array}$ & $\begin{array}{l}u_{\mathrm{tts}(\mathrm{t})} \\
(2,13)\end{array}$ & $\begin{array}{l}u_{\mathrm{tts}\left(\mathrm{g}^{+}\right)} \\
(2,14)\end{array}$ & $\begin{array}{l}u_{\mathrm{tts}\left(\mathrm{g}^{-}\right)} \\
(2,15)\end{array}$ & $\begin{array}{l}u_{\mathrm{ttg}^{-}(\mathrm{t})} \\
(2,16)\end{array}$ & $\begin{array}{l}u_{\mathrm{ttg}^{-}}\left(\mathrm{g}^{+}\right) \\
(2,17)\end{array}$ & $\begin{array}{l}u_{\mathrm{tg}^{-}\left(\mathrm{g}^{-}\right)} \\
(2,18)\end{array}$ \\
\hline $\begin{array}{l}u_{\mathrm{tg}^{+} \mathrm{t}(\mathrm{t})} \\
(3,19)\end{array}$ & $\begin{array}{l}\left.u_{\mathrm{tg}^{+}} \mathrm{tg}^{+}\right) \\
(3,20)\end{array}$ & $\begin{array}{l}u_{\mathrm{tg}^{+}} \mathrm{t}\left(\mathrm{g}^{-}\right) \\
(3,21)\end{array}$ & $\begin{array}{l}u_{\mathrm{tg}^{+} \mathrm{s}(\mathrm{t})} \\
(3,22)\end{array}$ & $\begin{array}{l}u_{\mathrm{tg}^{+}} \mathrm{s}\left(\mathrm{g}^{+}\right) \\
(3,23)\end{array}$ & $\begin{array}{l}u_{\mathrm{tg}^{+} \mathrm{s}\left(\mathrm{g}^{-}\right)} \\
(3,24)\end{array}$ & $\begin{array}{l}u_{\mathrm{tg}^{+} \mathrm{g}^{-}(\mathrm{t})} \\
(3,25)\end{array}$ & $\begin{array}{l}u_{\mathrm{tg}^{+} \mathrm{g}^{-}\left(\mathrm{g}^{+}\right)}(3,26)\end{array}$ & $\begin{array}{l}u_{\mathrm{tg}^{+} \mathrm{g}^{-}\left(\mathrm{g}^{-}\right)} \\
(3,27)\end{array}$ \\
\hline$u_{\mathrm{tg}^{-} \mathrm{t}(\mathrm{t})}$ & $\left.u_{\mathrm{tg}^{-}} \mathrm{t}_{\left(\mathrm{g}^{+}\right.}\right)$ & $u_{\mathrm{tg}^{-}} \mathrm{t}\left(\mathrm{g}^{-}\right)$ & $u_{\mathrm{tg}^{-} \mathrm{s}(\mathrm{t})}$ & $u_{\mathrm{tg}^{-} \mathrm{s}\left(\mathrm{g}^{+}\right)}$ & $u_{\mathrm{tg}^{-} \mathrm{s}\left(\mathrm{g}^{-}\right)}$ & $u_{\mathrm{tg}^{-} \mathrm{g}^{-}(\mathrm{t})}$ & $u_{\mathrm{tg}^{-}} \mathrm{g}^{-}\left(\mathrm{g}^{+}\right)$ & $u_{\mathrm{tg}^{-}} \mathrm{g}^{-}\left(\mathrm{g}^{-}\right)$ \\
\hline
\end{tabular}
hydroxybutyrate)

For the bond designation, see Figure 1 . The $U_{j}$ matrices of the first unit are

$$
\begin{gathered}
U_{1}=U_{\mathrm{a} 1}=C_{1} \otimes R_{1} \\
U_{\mathrm{b} 1}=C_{1} \otimes R_{3}
\end{gathered}
$$

and $U_{\mathrm{c} 1}$ of the $(S)$-form, $U_{\mathrm{c} 1}^{S}$, is a $3 \times 27$ matrix with the following elements: where $(l, m)(l=1-3$ and $m=1-27)$ represents the position in the matrix and, for example,

$$
u_{\mathrm{tg}^{+} \mathrm{s}\left(\mathrm{g}^{-}\right)}=\exp \left(-\Delta E_{\mathrm{tg}^{+}\left(\mathrm{g}^{-}\right)} / R T\right)
$$

is the statistical weight of the $\operatorname{tg}^{+} \mathrm{s}\left(\mathrm{g}^{-}\right)$conformation, in which bonds $\mathrm{a}_{1}, \mathrm{~b}_{1}, \mathrm{c}_{1}$ (main chain, see Figure. 1), and $d_{1}$ (side chain, in the parenthesis) adopt trans, gauche ${ }^{+}$, 
synperiplanar, and gauche ${ }^{-}$states, respectively. $E_{\mathrm{tg}^{+}\left(\mathrm{g}^{-}\right)}$is its conformational energy. The other elements and weights of the absent conformations are null.

The $U_{\mathrm{c} 1}$ matrix of the $(R)$-form, $U_{\mathrm{c} 1}^{R}$, can be derived from

$$
U_{\mathrm{c} 1}^{R}=Q_{3} U_{\mathrm{c} 1}^{S}\left(Q_{3} \otimes I_{3} \otimes Q_{3}\right)
$$

where

$$
Q_{3}=\left(\begin{array}{lll}
1 & 0 & 0 \\
0 & 0 & 1 \\
0 & 1 & 0
\end{array}\right)
$$

The geometrical parameters of the $(R)$-form are obtained from those of the $(S)$-form on the basis of the following correspondence between the two forms:

bonds 2 and a
$(S)$-form:
$\mathrm{t}$
$(R)$-form:
$\mathrm{t}$
bonds 3 and $b$
$(S)$-form:
$\mathrm{g}^{+} \quad \mathrm{g}^{-}$
$(R)$-form:
$\mathrm{g}^{-} \mathrm{g}^{+}$
bonds $4(7)$ and $\mathrm{c}(\mathrm{d})$
$(S)$-form:
$\begin{array}{lllllllll}\mathrm{t}(\mathrm{t}) & \mathrm{t}\left(\mathrm{g}^{+}\right) & \mathrm{t}\left(\mathrm{g}^{-}\right) & \mathrm{s}(\mathrm{t}) & \mathrm{s}\left(\mathrm{g}^{+}\right) & \mathrm{s}\left(\mathrm{g}^{-}\right) & \mathrm{g}^{-}(\mathrm{t}) & \mathrm{g}^{-}\left(\mathrm{g}^{+}\right) & \mathrm{g}^{-}\left(\mathrm{g}^{-}\right)\end{array}$
$(R)$-form:
$\begin{array}{llllllllll}\mathrm{t}(\mathrm{t}) & \mathrm{t}\left(\mathrm{g}^{-}\right) & \mathrm{t}\left(\mathrm{g}^{+}\right) & \mathrm{s}(\mathrm{t}) & \mathrm{s}\left(\mathrm{g}^{-}\right) & \mathrm{s}\left(\mathrm{g}^{+}\right) & \mathrm{g}^{+}(\mathrm{t}) & \mathrm{g}^{+}\left(\mathrm{g}^{-}\right) & \mathrm{g}^{+}\left(\mathrm{g}^{+}\right)\end{array}$

with the sign of the dihedral angle being switched $\left(\phi_{R}=-\phi_{S}\right)$.

The $U_{j}$ matrices of the subsequent repeating unit can be formulated as follows:

$$
\begin{gathered}
U_{\mathrm{a}}=C_{3} \otimes I_{3} \otimes I_{3} \\
U_{\mathrm{b}}=C_{3} \otimes C_{3} \otimes R_{3} \\
U_{\mathrm{c}}=U_{\mathrm{c} 1}
\end{gathered}
$$

For the terminal end, the $U_{\mathrm{m}}$ and $U_{\mathrm{n}}$ matrices are defined as

$$
\begin{aligned}
& U_{\mathrm{m}}=U_{a} \\
& U_{\mathrm{n}}=U_{b}
\end{aligned}
$$


Table S1. Geometrical parameters of PGA, used in the RIS calculations ${ }^{\text {a }}$

\begin{tabular}{|c|c|c|c|c|c|}
\hline \multicolumn{3}{|c|}{$\frac{\text { conformation }}{\text { bond }}$} & \multicolumn{2}{|c|}{ Bond a } & \multirow[b]{2}{*}{$\phi_{\mathrm{a}}$} \\
\hline c & $\mathrm{a}$ & $\mathrm{b}$ & $l_{\mathrm{a}}$ & $\angle \mathrm{a} \wedge \mathrm{b}$ & \\
\hline $\mathrm{t}$ & $\mathrm{t}$ & $\mathrm{t}$ & 1.347 & 115.6 & 0.0 \\
\hline $\mathrm{t}$ & $\mathrm{t}$ & $\mathrm{g}^{+}$ & 1.354 & 116.1 & -5.5 \\
\hline $\mathrm{t}$ & $\mathrm{t}$ & $\mathrm{g}^{-}$ & 1.354 & 116.1 & 5.5 \\
\hline $\mathrm{s}$ & $\mathrm{t}$ & $\mathrm{t}$ & 1.341 & 115.7 & 0.0 \\
\hline s & $\mathrm{t}$ & $\mathrm{g}^{+}$ & 1.346 & 115.6 & -3.1 \\
\hline s & $\mathrm{t}$ & $\mathrm{g}^{-}$ & 1.346 & 115.6 & 3.1 \\
\hline
\end{tabular}

\begin{tabular}{ccccrr}
\multicolumn{7}{c}{ Bond b } \\
\cline { 1 - 2 } conformation & & & \\
$\mathrm{a}$ & $\mathrm{b}$ & $\mathrm{c}$ & $l_{\mathrm{b}}$ & $\angle \mathrm{b} \wedge \mathrm{c}$ & \multicolumn{1}{c}{$\phi_{\mathrm{b}}$} \\
& & & & & \\
$\mathrm{t}$ & $\mathrm{t}$ & $\mathrm{t}$ & 1.426 & 108.5 & 0.0 \\
$\mathrm{t}$ & $\mathrm{g}^{+}$ & $\mathrm{t}$ & 1.423 & 111.1 & 98,2 \\
$\mathrm{t}$ & $\mathrm{g}^{-}$ & $\mathrm{t}$ & 1.423 & 111.1 & $-98,2$ \\
$\mathrm{t}$ & $\mathrm{t}$ & $\mathrm{s}$ & 1.432 & 111.8 & 0.0 \\
$\mathrm{t}$ & $\mathrm{g}^{+}$ & $\mathrm{s}$ & 1.429 & 115.0 & 97.7 \\
$\mathrm{t}$ & $\mathrm{g}^{-}$ & $\mathrm{s}$ & 1.429 & 115.0 & -97.7
\end{tabular}

Bond c

\begin{tabular}{|c|c|c|c|c|c|}
\hline \multicolumn{3}{|c|}{ conformatior } & \multirow[b]{2}{*}{$l_{\mathrm{c}}$} & \multirow[b]{2}{*}{$\angle \mathrm{c} \wedge \mathrm{a}$} & \multirow[b]{2}{*}{$\phi_{\mathrm{c}}$} \\
\hline b & $\mathrm{c}$ & $\mathrm{a}$ & & & \\
\hline $\mathrm{t}$ & $\mathrm{t}$ & $\mathrm{t}$ & 1.516 & 108.7 & 0.0 \\
\hline $\mathrm{t}$ & $\mathrm{s}$ & $\mathrm{t}$ & 1.514 & 114.4 & 180.0 \\
\hline $\mathrm{g}^{+}$ & $\mathrm{t}$ & $\mathrm{t}$ & 1.521 & 109.5 & 1.0 \\
\hline $\mathrm{g}^{+}$ & $\mathrm{s}$ & $\mathrm{t}$ & 1.520 & 113.5 & 179.9 \\
\hline $\mathrm{g}^{-}$ & $\mathrm{t}$ & $\mathrm{t}$ & 1.521 & 109.5 & -1.0 \\
\hline $\mathrm{g}^{-}$ & $\mathrm{s}$ & $\mathrm{t}$ & 1.520 & 113.5 & -179.9 \\
\hline
\end{tabular}

${ }^{a}$ Obtained from the structural optimization for the dimer, 2-methoxy-2-oxoethyl 2-acetoxyacetate (MOAA), at the B3LYP/6-311+G(2d,p) level. Only the existent conformations are listed. Symbols: $\mathrm{t}$, trans; g, gauche; $\mathrm{s}$, synperiplanar; $l_{\mathrm{a}}$, length of bond $\mathrm{a} ; \angle \mathrm{a} \wedge \mathrm{b}$, angle between bonds $\mathrm{a}$ and $\mathrm{b} ; \phi_{\mathrm{a}}$, dihedral angle of bond $\mathrm{a}$. The dihedral angle is defined according to the tradition of the RIS scheme: $\phi_{\mathrm{t}} \sim 0^{\circ}, \phi_{\mathrm{g} \pm} \sim \pm 120^{\circ}$, and $\phi_{\mathrm{s}} \sim \pm 180^{\circ}$. 
Table S2. Geometrical Parameters of $(S)$ Form of P2HB, Used in the RIS Calculations ${ }^{a}$

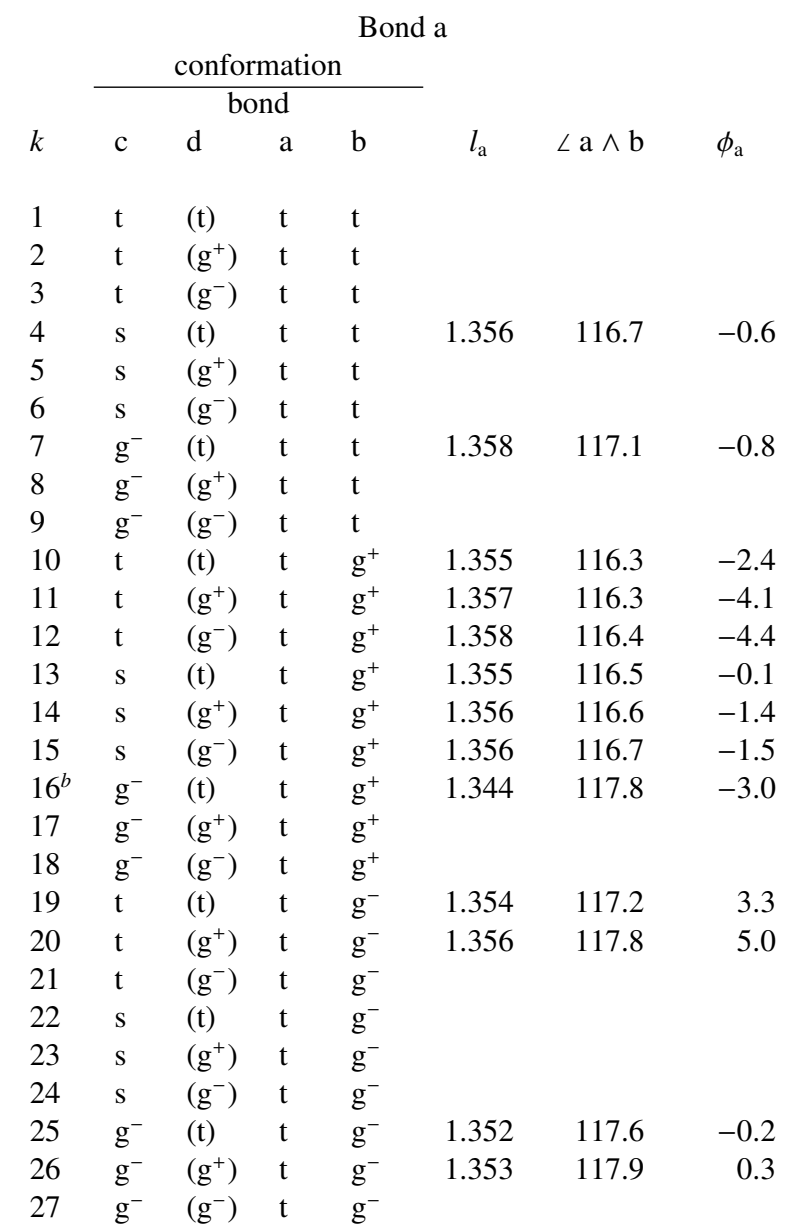

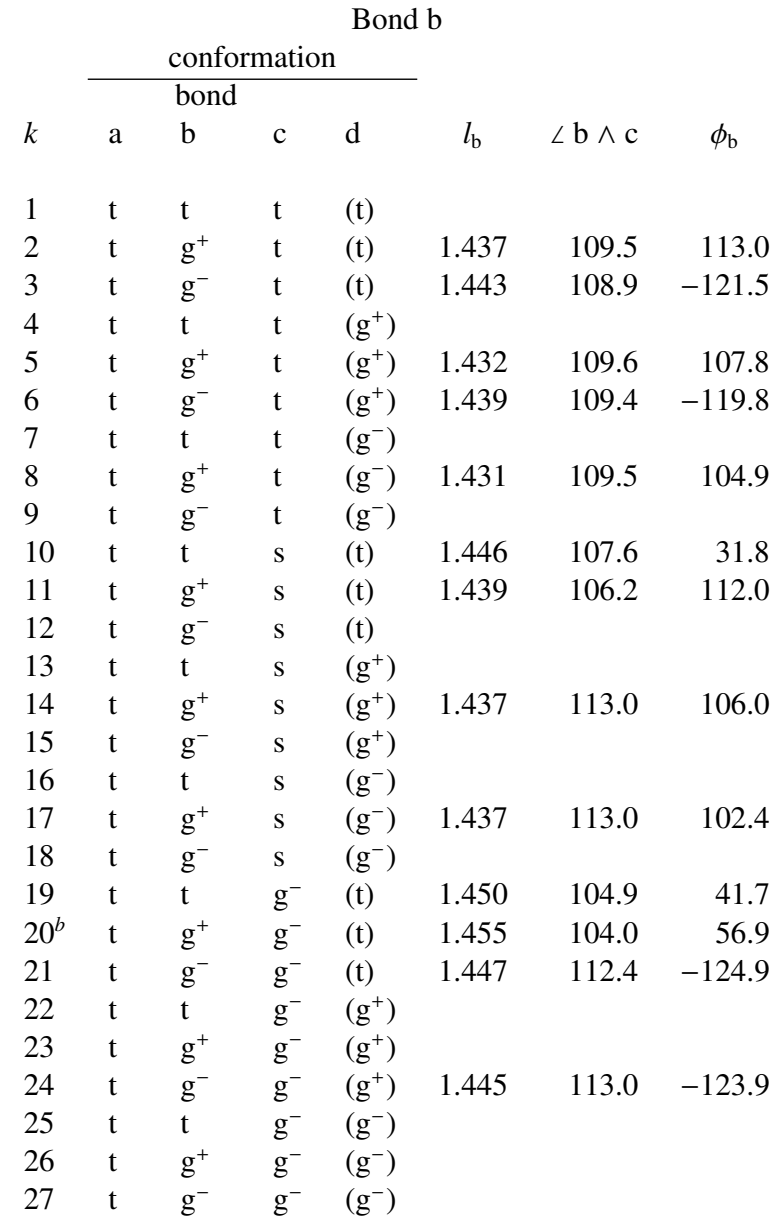

Bond c conformation

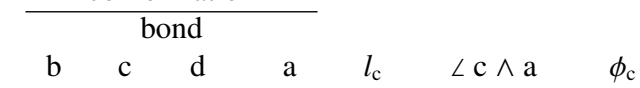

$\begin{array}{llllllll}1 & \mathrm{t} & \mathrm{t} & (\mathrm{t}) & \mathrm{t} & & & \\ 2 & \mathrm{t} & \mathrm{t} & \left(\mathrm{g}^{+}\right) & \mathrm{t} & & & \\ 3 & \mathrm{t} & \mathrm{t} & \left(\mathrm{g}^{-}\right) & \mathrm{t} & & & \\ 4 & \mathrm{t} & \mathrm{s} & (\mathrm{t}) & \mathrm{t} & 1.524 & 112.4 & 131.9 \\ 5 & \mathrm{t} & \mathrm{s} & \left(\mathrm{g}^{+}\right) & \mathrm{t} & & & \\ 6 & \mathrm{t} & \mathrm{s} & \left(\mathrm{g}^{-}\right) & \mathrm{t} & & & \\ 7 & \mathrm{t} & \mathrm{g}^{-} & (\mathrm{t}) & \mathrm{t} & 1.529 & 110.7 & -109.2 \\ 8 & \mathrm{t} & \mathrm{g}^{-} & \left(\mathrm{g}^{+}\right) & \mathrm{t} & & & \\ 9 & \mathrm{t} & \mathrm{g}^{-} & \left(\mathrm{g}^{-}\right) & \mathrm{t} & & & \\ 10 & \mathrm{~g}^{+} & \mathrm{t} & (\mathrm{t}) & \mathrm{t} & 1.529 & 110.7 & -30.1 \\ 11 & \mathrm{~g}^{+} & \mathrm{t} & \left(\mathrm{g}^{+}\right) & \mathrm{t} & 1.528 & 110.0 & -17.0 \\ 12 & \mathrm{~g}^{+} & \mathrm{t} & \left(\mathrm{g}^{-}\right) & \mathrm{t} & 1.529 & 109.8 & -10.7 \\ 13 & \mathrm{~g}^{+} & \mathrm{s} & (\mathrm{t}) & \mathrm{t} & 1.526 & 113.0 & 155.0 \\ 14 & \mathrm{~g}^{+} & \mathrm{s} & \left(\mathrm{g}^{+}\right) & \mathrm{t} & 1.526 & 113.6 & 166.1 \\ 15 & \mathrm{~g}^{+} & \mathrm{s} & \left(\mathrm{g}^{-}\right) & \mathrm{t} & 1.528 & 113.8 & 173.2 \\ 16^{b} & \mathrm{~g}^{+} & \mathrm{g}^{-} & (\mathrm{t}) & \mathrm{t} & 1.531 & 110.6 & -92.1 \\ 17 & \mathrm{~g}^{+} & \mathrm{g}^{-} & \left(\mathrm{g}^{+}\right) & \mathrm{t} & & & \\ 18 & \mathrm{~g}^{+} & \mathrm{g}^{-} & \left(\mathrm{g}^{-}\right) & \mathrm{t} & & & \\ 19 & \mathrm{~g}^{-} & \mathrm{t} & (\mathrm{t}) & \mathrm{t} & 1.536 & 112.2 & 30.3 \\ 20 & \mathrm{~g}^{-} & \mathrm{t} & \left(\mathrm{g}^{+}\right) & \mathrm{t} & 1.534 & 111.4 & 19.6 \\ 21 & \mathrm{~g}^{-} & \mathrm{t} & \left(\mathrm{g}^{-}\right) & \mathrm{t} & & & \\ 22 & \mathrm{~g}^{-} & \mathrm{s} & (\mathrm{t}) & \mathrm{t} & & & \\ 23 & \mathrm{~g}^{-} & \mathrm{s} & \left(\mathrm{g}^{+}\right) & \mathrm{t} & & & \\ 24 & \mathrm{~g}^{-} & \mathrm{s} & \left(\mathrm{g}^{-}\right) & \mathrm{t} & & & \\ 25 & \mathrm{~g}^{-} & \mathrm{g} & (\mathrm{t}) & \mathrm{t} & 1.532 & 111.6 & -143.6 \\ 26 & \mathrm{~g}^{-} & \mathrm{g}^{-} & \left(\mathrm{g}^{+}\right) & \mathrm{t} & 1.531 & 112.0 & -148.8 \\ 27 & \mathrm{~g}^{-} & \mathrm{g}^{-} & \left(\mathrm{g}^{-}\right) & \mathrm{t} & & & \\ & & & & & & & \end{array}$

${ }^{a}$ Obtained from the geometrical optimization for the monomer, methyl $(S)$-2-acetoxybutanoate ((S)-MAB), at the B3LYP/6-311+G(2d,p) level. The geometrical parameters of the $(R)$-form were derived from the above data by the symmetry operation as described in Formulation B. The blank line represents the absence of the conformation. Symbols: $\mathrm{t}$, trans; g, gauche; s, synperiplanar; $l_{\mathrm{a}}$, length of bond a; $\angle a \wedge b$, angle between bonds a and $\mathrm{b} ; \phi_{\mathrm{a}}$, dihedral angle of bond a. The dihedral angle is defined according to the tradition of the RIS scheme: $\phi_{\mathrm{t}} \sim 0^{\circ}, \phi_{\mathrm{g} \pm} \sim \pm 120^{\circ}$, and $\phi_{\mathrm{s}} \sim \pm 180^{\circ} .{ }^{b}$ Only the data on this conformation were obtained from the dimer, $(S)$-1-methoxy-1-oxobutan-2-yl $(S)$ 2-acetoxybutanoate $((S, S)$-MOAB $)$. 
Table S3. Atomic Born Charge Tensors of PGA Crystal

\begin{tabular}{|c|c|c|c|}
\hline $\mathrm{CH}_{2}$ & $e=0.422624$ & & \\
\hline & 1 & 2 & 3 \\
\hline 1 & $9.1022 \times 10^{-2}$ & 0.0000 & $-2.3575 \times 10^{-1}$ \\
\hline 2 & 0.0000 & $2.8805 \times 10^{-1}$ & 0.0000 \\
\hline 3 & $-5.3065 \times 10^{-1}$ & 0.0000 & $8.8880 \times 10^{-1}$ \\
\hline $\mathrm{C}=\mathrm{O}$ & $e=1.626448$ & & \\
\hline & 1 & 2 & 3 \\
\hline 1 & 2.0587 & 0.0000 & $1.7159 \times 10^{-1}$ \\
\hline 2 & 0.0000 & $5.1698 \times 10^{-1}$ & 0.0000 \\
\hline 3 & $6.6043 \times 10^{-1}$ & 0.0000 & 2.3037 \\
\hline O- & $e=-1.195174$ & & \\
\hline & 1 & 2 & 3 \\
\hline 1 & $-9.0496 \times 10^{-1}$ & 0.0000 & $-3.0217 \times 10^{-1}$ \\
\hline 2 & 0.0000 & $-4.8247 \times 10^{-1}$ & 0.0000 \\
\hline 3 & $-2.5624 \times 10^{-1}$ & 0.0000 & -2.1981 \\
\hline $\mathrm{C}=\underline{\mathrm{O}}$ & $e=-1.029478$ & & \\
\hline & 1 & 2 & 3 \\
\hline 1 & -1.5687 & 0.0000 & $5.0230 \times 10^{-2}$ \\
\hline 2 & 0.0000 & $-5.8903 \times 10^{-1}$ & 0.0000 \\
\hline 3 & $-3.6104 \times 10^{-1}$ & 0.0000 & $-9.3073 \times 10^{-1}$ \\
\hline $\mathrm{C}_{2}$ & $e=0.087791$ & & \\
\hline & 1 & 2 & 3 \\
\hline 1 & $1.6198 \times 10^{-1}$ & $1.9728 \times 10^{-2}$ & $-4.7644 \times 10^{-2}$ \\
\hline 2 & $4.4763 \times 10^{-3}$ & $1.3324 \times 10^{-1}$ & $-1.8916 \times 10^{-2}$ \\
\hline 3 & $5.7350 \times 10^{-2}$ & $1.6491 \times 10^{-2}$ & $-3.1847 \times 10^{-2}$ \\
\hline
\end{tabular}

${ }^{a}$ In the unit of elementary charge. 
Table S4. Internal Energies of Representative (Stable) Conformers of ( $S$ )-MAB and $(S, S)$-MOAB

$\Delta E_{k}\left(\mathrm{kcal} \mathrm{mol}^{-1}\right)$

\begin{tabular}{|c|c|c|c|c|c|c|c|c|c|}
\hline \multicolumn{4}{|c|}{ bond } & \multicolumn{3}{|c|}{ gas } & \multicolumn{3}{|c|}{ DMSO } \\
\hline $\mathrm{a}$ & $\mathrm{b}$ & $\mathrm{c}$ & d & $(S)-\mathrm{MAB}^{a}$ & $(S, S)-\mathrm{MOAB}^{b}$ & $(S, S)-\mathrm{MOAB}^{c}$ & $(S)-\mathrm{MAB}^{a}$ & $(S, S)-\mathrm{MOAB}^{b}$ & $(S, S)-\mathrm{MOAB}^{c}$ \\
\hline $\mathrm{t}$ & $\mathrm{g}^{+}$ & $\mathrm{t}$ & $(\mathrm{t})$ & 0.00 & 0.00 & 0.00 & 0.00 & 0.00 & 0.00 \\
\hline $\mathrm{t}$ & $\mathrm{g}^{+}$ & $\mathrm{t}$ & $\left(\mathrm{g}^{+}\right)$ & -0.68 & -0.79 & -0.76 & -0.48 & -0.56 & -0.55 \\
\hline $\mathrm{t}$ & $\mathrm{g}^{+}$ & $\mathrm{t}$ & $\left(\mathrm{g}^{-}\right)$ & -0.67 & -0.73 & -0.71 & -0.37 & -0.47 & -0.47 \\
\hline $\mathrm{t}$ & $\mathrm{g}^{+}$ & $\mathrm{s}$ & (t) & 0.42 & 0.49 & 0.77 & 0.50 & 0.41 & 0.58 \\
\hline $\mathrm{t}$ & $\mathrm{g}^{+}$ & $\mathrm{s}$ & $\left(\mathrm{g}^{+}\right)$ & -0.15 & -0.17 & 0.12 & 0.05 & -0.15 & 0.03 \\
\hline $\mathrm{t}$ & $\mathrm{g}^{+}$ & $\mathrm{s}$ & $\left(\mathrm{g}^{-}\right)$ & -0.10 & -0.12 & 0.23 & 0.22 & -0.02 & 0.22 \\
\hline
\end{tabular}

${ }^{a}$ As calculated. ${ }^{b}$ As calculated with bonds a and $\mathrm{b}$ set in $\mathrm{t}$ and $\mathrm{g}^{+}$states, respectively. ${ }^{c}$ Averaged according to eq 1 . 